\title{
Analysis of Data about Material Tests Based on EMD Method
}

\author{
http://dx.doi.org/10.3991/ijoe.v9iS6.2800 \\ Kai Peng \\ Wuhan University of Technology, Wuhan, China
}

\begin{abstract}
Using EMD signal decomposition method to decompose the data of material test and comparing the difference of Hilbert spectrum in signal components on the different stages in the paper,the amplitude and frequency variation in the test process is calculted so as to determine the strength and reliability of the material as well as from the obtained experience to judge the health of the material used. It innovatively applies the segment data to make comparisons, accurately locates the specific data segment with structural damage and provides the simutaneous monitoring scheme while reducing the amount of calculation.
\end{abstract}

Index Terms-HHT, EMD, Hilbert spectrum, Aimf, Fimf

\section{INTRODUCTION}

People can reliably learn whether the health condition of the material is good or not and obtain features of its structure behavior by excavating and analyzing the data achieved in daily monitoring. Using parameters of structural vibration response and systematic dynamic behavior to detect structural damage is a hot topic of domestic and foreign researches; and a number of research methods have also been proposed ${ }^{[1]}$.

Hilbert Huang Transform (HHT) is an internationally novel method of digital signal processing, whose data handling capacity remedies the shortages of traditional data processing methods like Fourier transform and wavelet analysis. We can describe the health condition of the structure more accurately and more clearly by applying the HHT method to analyze data about system monitoring.

According to basic thoughts of HHT, all collected data are composed of basic signals, i.e., intrinsic mode function (IMF), the mutual superposition of IMFs forms composite signals and the IMF of data signals, which is obtained by the decomposition method of empirical mode is the 'base' generated by self-adaption. In accordance with the 'base' decomposed and obtained by the HHT method, original data about the monitoring of the research material can be applied to parameter identification of dynamic property, signal decomposition and reconstruction, damage identification, signal denoising and analysis of long-period tendency etc.

This thesis uses the HHT method to analyze a group of data about structural tests, utilizes the concept of degree of nonlinearity, gives results of structural damage tests in monitoring data and proposes guidance and suggestions for structural health monitoring in practical use.

\section{BASIC THINKING ON THE APPLICATION OF THE HHT METHOD TO THE DATA ANALYSIS OF TESTS}

HHT (Hilbert-Huang Transform) proposed by American Chinese N. E. Huang et al. in 1998 is a new method of data analysis.

HHT can handle non-linear and non-stationary signals. N. E. Huang et al. deem any signal is composed of basic signals, i.e., intrinsic mode signal or intrinsic mode function (IMF), and the mutual superposition of IMFs forms composite signals.

Compared with traditional signal or data processing methods, such as Fourier transform, short-time Fourier transform and Wavelet Transform(WT), HHT are featured by the following aspects.

(1)HHT can analyze non-linear and non-stationary signals.

(2)HHT holds complete self-adaptation.

(3)HHT is not restricted by Heisenberg uncertainty principle.

(4)The instantaneous frequency of HHT is obtained by derivation.

With all IMF components obtained by using the HHT method to carry out EMD decomposition for signals based on the foregoing features, the information about the instantaneous state of the function can be achieved.

The amplitude function :

$$
\begin{gathered}
a(t)=\sqrt{s^{2}(t)+H^{2}[s(t)]}, \\
\omega(t)=d \phi(t) / d t, f(t)=\omega(t) / 2 \pi .
\end{gathered}
$$

The instantaneous frequency and the instantaneous angular frequency are

(2)

$$
\omega(t)=d \phi(t) / d t \quad \text { and } \quad f(t)=\omega(t) / 2 \pi \quad .
$$

The frequency of the intrinsic mode function (Fimf) is

$$
\text { Fimf }=\frac{1}{T} \int_{0}^{T} f(t) d t .
$$

The amplitude of the intrinsic mode function (Aimf) is

(4)

$$
\operatorname{Aimf}=\frac{1}{T} \int_{0}^{T} a(t) d t
$$

Use Hilbert spectrum t realize

$$
\operatorname{Aimf}=\int_{\omega_{1}}^{\omega_{2}} h(\omega) d \omega
$$


(5)

$$
F i m f=\frac{1}{2 \pi \operatorname{Aimf}} \int_{\omega_{1}}^{\omega_{2}} \omega h(\omega) d \omega
$$

As tests have been repeated many times, the frequency of the intrinsic mode function (Fimf) and the amplitude of the intrinsic mode function (Aimf), which are corresponding to the data at the initial stage can be obtained by selecting a group of data at the initial stage of the experiment for EMD decomposition. Compare them with the Fimf and Aimf of the data with the same length at the later stage of the experiment. If obvious changes appear, it will state that the structure gives different feedback to the same motivation, structural damage has existed and we can find how many times the experiment has been carried out when the structural damage begins to occur by limited-time screening. This is of great value for the analysis of experimental results.

To learn the change in the two values visually, we can use relative variables to measure them.

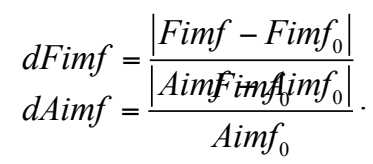

Define the health interval of dFimf and dAimf according to practical experience. When the calculated dFimf and dAimf stays in the interval $(0, \varepsilon)$, it indicates the structure is still healthy. If dFimf and dAimf stays in $[\varepsilon, M)$, it will suggest that the structure has had damage.

\section{ANALYSIS OF EXAMPLES ABOUT TEST DATA}

The experiment records 3 million test loading data and a part of them are selected and shown in the following figure.

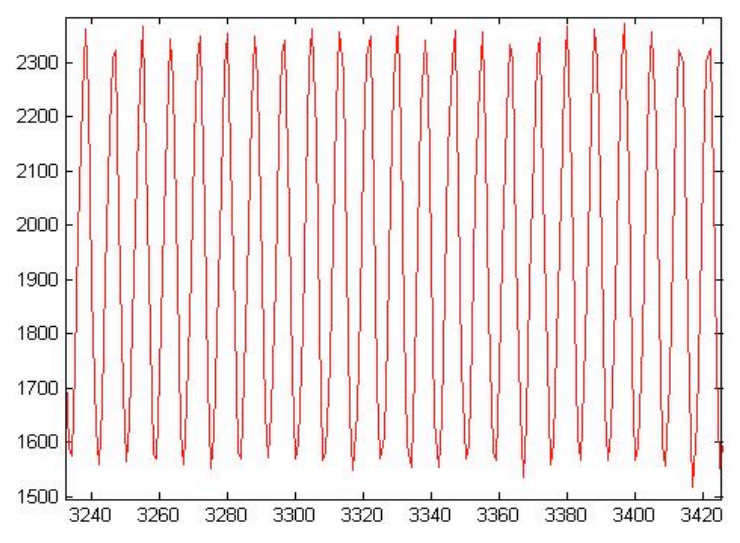

Fig. 1. Part of the data dapture.

Select the data at the initial stage as a reference standard of healthy structure to calculate the value of Fimf and Aimf.

The selected reference data are from the 50001st to the 60000th.

Carry out EMD decomposition, whose results are shown in the following figure.

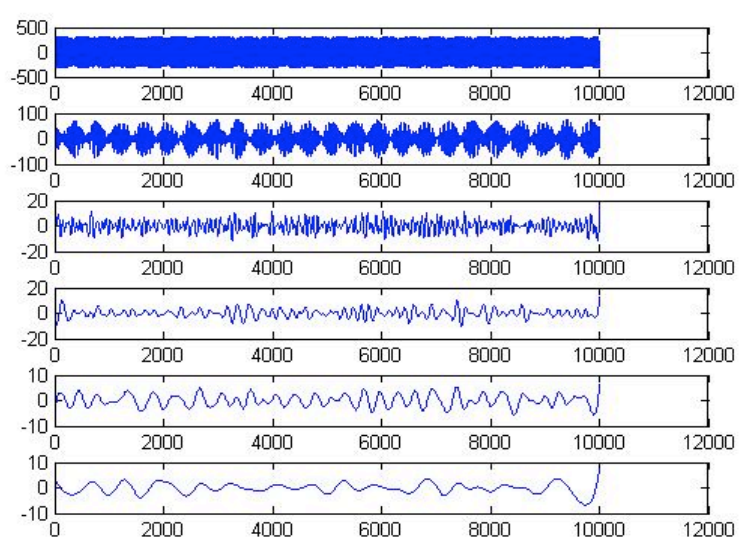

Fig. 2. IMFS1-6

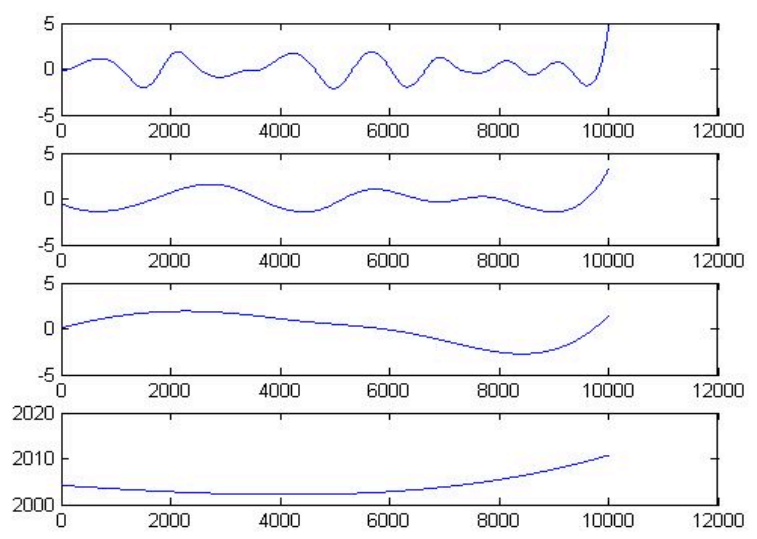

Fig. 3. IMFS7-10

The image of the marginal spectrum is shown in the following figure.

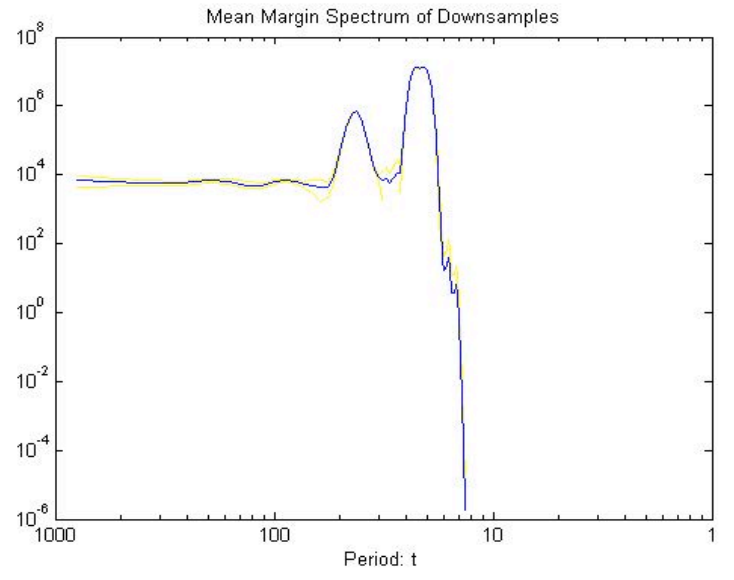

Fig. 4. Initial marginal spectrum.

The values of Fimf and Aimf are $24.98 \mathrm{~Hz}$ and $0.225 \mathrm{~m} / \mathrm{s} 2$.

Then, select data to compare with the data of the healthy structure. The selected reference data is from the 1050001 st to the 1060000th.

Carry out EMD decomposition, whose results are shown in the following figure. 


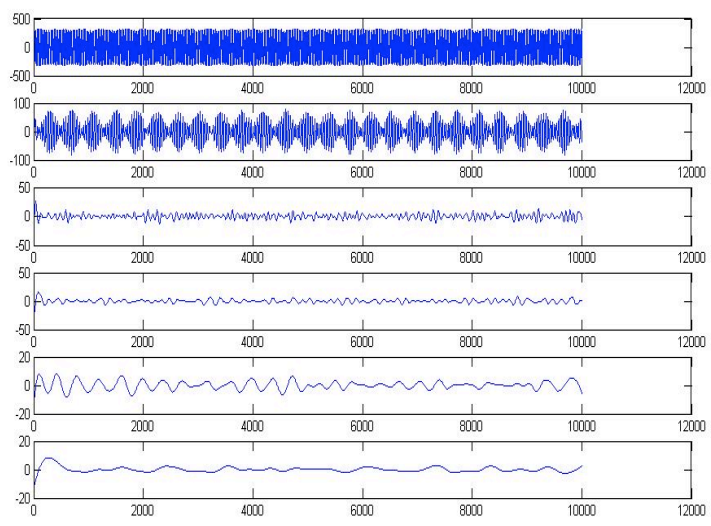

Fig. 5. IMFS1-6

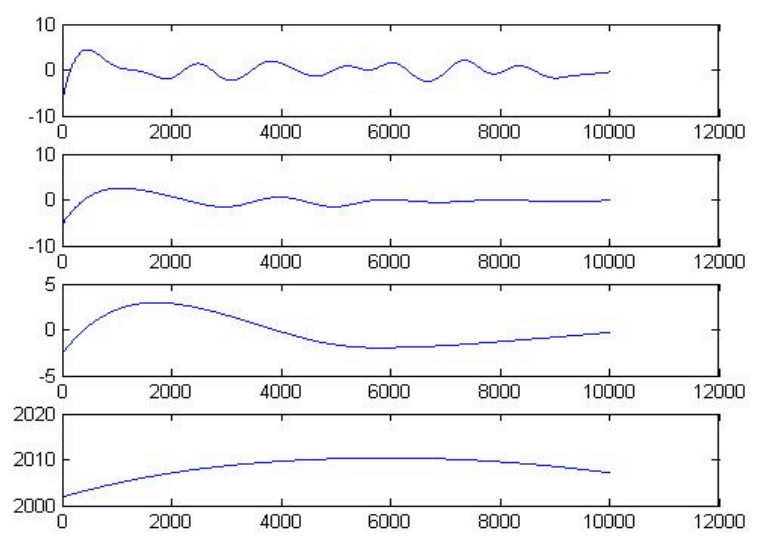

Fig. 6. IMFS7-10

The marginal spectrum is shown in the following figure.

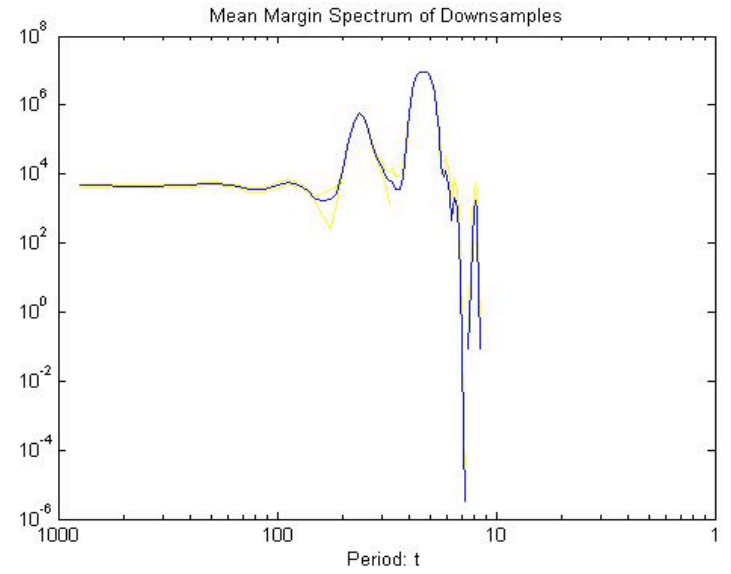

Fig. 7. The end marginal spectrum.

The values of Fimf and Aimf are $22.95 \mathrm{~Hz}$ and $0.205 \mathrm{~m} / \mathrm{s} 2$.

$$
\begin{aligned}
& d \text { Fimf }=\frac{\mid \operatorname{Fimf}_{-}-\text {Fimf }_{0} \mid}{\operatorname{Fimf}_{0}}=8.13 \% \\
& d A \operatorname{Aimf}=\frac{\left|\operatorname{Aimf}-\operatorname{Aimf}_{0}\right|}{\operatorname{Aimf}_{0}}=8.9 \% .
\end{aligned}
$$

Then, select ending data to compare with the data of the healthy structure. The selected reference data is from the 2950001 st to the 2960000th.

Similarly, carry out EMD decomposition.

The marginal spectrum is shown in the following figure.

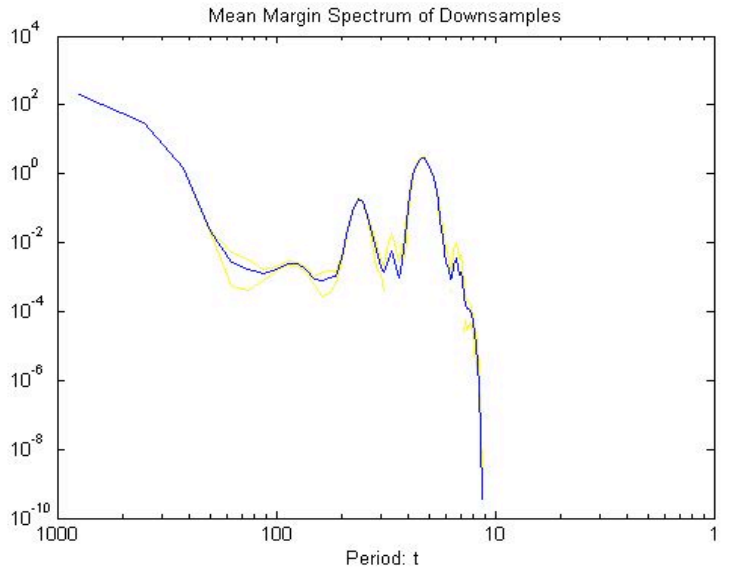

Fig. 8. Terminal marginal spectrum.

The values of Fimf and Aimf are $17.05 \mathrm{~Hz}$ and $0.165 \mathrm{~m} / \mathrm{s} 2$.

$$
\begin{aligned}
& d F i m f=\frac{\mid \text { Fimf }- \text { Fimf }_{0} \mid}{\text { Fimf }_{0}}=30.17 \% \\
& d \operatorname{Aimf}=\frac{\left|\operatorname{Aimf}-\operatorname{Aimf}_{0}\right|}{\operatorname{Aimf}_{0}}=26.7 \% \text {. }
\end{aligned}
$$

If define the health interval of $\operatorname{dAimf}=\frac{\left|\operatorname{Aimf}-\operatorname{Aimf} f_{0}\right|}{\operatorname{Aimf} f_{0}}$ is $(0,0.3)$, it will be found that the 2610000th data just stays in the interval by the test. In another word, the material has no damage in 2610000 fatigue tests.

\section{CONCLUSION}

Using the processing capacity of EMD for nonstationary and non-linear process data and adopting the concept of Fimf and Aimf can not only analyze the data obtained in fatigue tests effectively but also quantize the health degree of the structure by relative values dFimf and dAimf. According to practical experience, we can define the range of the health degree to help engineers give valuable judgment in practical monitoring of structural health.

\section{ACKNOWLEDGMENT}

Finally, I would like to thank the Fundamental Research Funds for the Central Universities (2012-Ia-030) and The State Oceanic Administration Laboratory Open Fund (LDAA-2012-01) for providing funds to support this research.

\section{REFERENCES}

[1] J. N. Yang, Y. Lei, S. Lin, and N. Huang, "Hilbert-Huang Based Approach for Structural Damage Detection," Journal of Engineering Mechanics, vol. 130, pp.85-95, 2004. http://dx.doi.org/10.1061/(ASCE)0733-9399(2004)130:1(85)

[2] H. Sohn, C. R. Farrar, and F. M. Hemez, A Review of Structural Health Monitoring Literature: 1996-2001, Los Alamos National Laboratory Report, LA-13976-MS, 2004.

[3] N. E. Huang, Z. Shen, and S. R. Long, "The Empirical Mode Decomposition and Hilbert Spectrum for Nonlinear and Nonstationary Time Series Analysis," Proc. Royal Society of London Series, pp.903-995, 1998.

[4] C. C. Chih and W. P. Chun, "Dynamic Characterization of a Damaged Beam Using Empirical Mode Decomposition and Hilbert Spectrum method," Health Monitoring and Smart 


\section{AnAlysis of DATA ABOUt MATERIAL TESTS BASED ON EMD METHOD}

Nondestructive Evaluation of Structural and Biological Systems III, Proc. Of. SPIE, pp.399-410, 2004.

[5] Y. L. Xu and J. Chen, "Structural Damage Detection Using Empirical Mode Decomposition: Experimental Investigation," Journal of Engineering Mechanics, vol. 130, Issue 11, pp. 12791288, 2004. http://dx.doi.org/10.1061/(ASCE)0733-9399(2004) 130:11(1279)

[6] Z. Nie, H. Hao, and H. Ma, "Structural Damage Detection Using Phase Space Geometry Changes," in Proceedings of 4th International Conference on Experimental Vibration Analysis for Civil Engineering Structures, Varenna, Italy, pp.407-416, 2011.

[7] S. Torkamani, E. A. Butcher, M. D. Todd, and G. Park, "Detection of System Changes due to Damage Using A Tuned Hyperchaotic Probe," Smart Materials and Structures, Vol. 20, Issue 2, pp. 1-16, 2011.

[8] Q. X. ZHANG, Z. D. DUAN, and L. Jankowski, "Experimental Validation of A Fast Dynamic Load Identification Method Based on Load Shape Function," Journal of Vibration and Shock, Vol. 30, Issue 9, pp. 105-110, 2011.

[9] K. Zhang, H. Li, and Z. Duan, A Probabilistic Damage Identification Approach for Structures with Uncertainties under Unknown Input," Mechanical Systems and Signal Processing, Vol.
25, Issue 4, pp. 1126-1145, 2011. http://dx.doi.org/10.1016/j.ymssp.2010.10.017

[10] Q. Zhang, L. Jankowski, and Z. Duan. "Identification of Coexistent Load and Damage," Structural Multidisciplinary Optimization, Vol. 41, Issue 2, pp. 243-253, 2010. http://dx.doi.org/10.1007/s00158-009-0421-1

[11] Q. Zhang, L. Jankowski, and Z. Duan, "Simultaneous Identification of Moving Masses and Structural Damage," Structural Multidisciplinary Optimization, Vol. 42, Issue 6, pp. 907-922, 2010. http://dx.doi.org/10.1007/s00158-010-0528-4

\section{AUTHORS}

Kai Peng is with the School of Science, Wuhan University of Technology, Wuhan, Hubei 430072 China (e-mail: pengkaiwh@vip.qq.com).

This article is an extended and modified version of a paper presented at the 2012 International Conference on Artificial Intelligence and Its Application in Industry Production (AIAIP 2012), held in Wuhan, China in December 2012. Manuscript received 19 May 2013. Published as resubmitted by the authors 26 June 2013 . 